\title{
OPEN
}

\section{1 \\ Meeting People Is Easy: The Pragmatics of Interdisciplinary Collaboration}

\begin{abstract}
This chapter establishes the pragmatics of how we have actually put together specific moments of interdisciplinary collaboration. Thinking through the 'why' of interdisciplinarity (and drawing on some of our collaborators' own accounts), it situates our own work within a particular interdisciplinary constellation of institutions and endeavours. The chapter offers some practical advice for getting projects started and funded - and, drawing on some peer review material, pays particular attention to the labours of interdisciplinary publication. It concludes with a call for interdisciplinary collaborators to treat their own progress as a legitimate research object, and offers some advice for tracking that progress.
\end{abstract}

Keywords: advice; collaboration; interdisciplinarity; neuroscience; social science

Callard, Felicity and Des Fitzgerald. Rethinking Interdisciplinarity across the Social Sciences and Neurosciences. Basingstoke: Palgrave Macmillan, 2015. DOI: $10.1057 / 9781137407962.0004$. 


\section{Introduction}

In developing an account of the pragmatics of collaboration, this chapter is aimed at those who might welcome some concrete suggestions for embarking on - or persisting with - interdisciplinary research. In offering such an account, we will resist normative claims on what the reader should do; rather, we bring to the foreground some of the resources, motivations, infrastructures, frameworks, people, and modes of working that enabled (and, undoubtedly, constrained) the paths we have ourselves taken. Many of these entities will reappear, episodically, as the book threads its conceptual arguments through different research studies, workshops, and collaborations; we want to orient the reader at the start, so that she can carry some of these details with her as she proceeds through later chapters.

First, let us note that the book emerges out of a configuration of funding and network(ing) opportunities that we believe to be somewhat particular. From 2008 to 2015 (the stretch of time during which the two of us have been engaged in interdisciplinary research across the social sciences and neurosciences), certain kinds of activities and collaborations across the terrain of the mind and brain became possible, we believe, because of particular configurations and pressures - epistemological, institutional, and disciplinary. To paint in very broad strokes: these opportunities came in the wake of (and, in some way, the disappointments of) the 'Decade of the Brain', on the one hand, and the Human Genome Project, on the other (see e.g. Meloni 2014a), around which a tacit intellectual and administrative conviction emerged that the neurosciences and the social sciences (among other sets of actors) needed to find some kind of shared language, and, through such language, to work better together. (Here, in passing, is a potential historical or sociological research project that we ourselves do not attempt: are we right in diagnosing an interesting 'moment' in interdisciplinary collaborations across the neurosciences and social sciences at this particular point? And if we are, how should one account for its emergence? There are, after all, many reasons why inter- or cross-disciplinary assemblages gain energy: David Engerman, to take one example, has stressed the importance not of research imperatives but of pedagogy (via both undergraduate and graduate training) in understanding the rise of interdisciplinary 'area studies' after the Second World War (Engerman 2015)). One of the core tasks of this chapter, then, is to bring that moment into visibility - to use our own experiences to 
begin to understand, and to diffract, a broader neuro/social/science landscape that has emerged in the last decade or so.

In this sense, rather than focusing on providing a quickly dated and extensive list of recommendations of specific places to go, and bodies to talk to, we place greater attention on how we progressed - with reference to some of the ideas, people, things, institutions, and funders that helped us along the way. Undoubtedly, there is now a whole range of new actors (both individual and institutional) of which we are barely even aware. Still, our hope is that the reader takes less note of where we have gone, with whom we have collaborated, or what exactly we have done, and pays attention instead to the kinds of moves - across epistemological and disciplinary boundaries, towards particular institutional and/or sub-disciplinary problematics - that have gotten us there. The chapter moves from general reflections on the motivations and structures that assist in opening up interdisciplinary collaboration, to the specific means through which we were able to build collaborations and garner expertise, to the strange world of interdisciplinary funding and peer review - and to how, finally, one might track the look and feel of interdisciplinary practice, such that that practice stops being a vaguely gestural thing 'out there', and becomes, instead, an object of scholarly attention in its own right.

\section{Why are you here?}

If you are reading this book, perhaps your appetite has been whetted whether recently or a long while ago - for interdisciplinary collaboration in the space enjoining the social sciences, the humanities and the natural sciences. Perhaps this has come via conferences or workshops you attended, or through journal articles or books you read (e.g. Slaby and Choudhury 2011). Perhaps your research interests have taken you, more or less unbidden, to a point of crossing between the sciences, social sciences and humanities - such as legal debates over the extent to which neuroscientific findings might be used in law courts (Jones et al. 2013). In any event, it is worth paying attention to what has led you to the question of interdisciplinary collaboration: not only might this help to diagnose what you might desire from it, but it might also help to shape where you would be happy - and unhappy - for any putative collaboration to take you. 
One of us (DF), for example, is here, ultimately, because of an argument in a graduate seminar, many years ago, about whether or not schizophrenia could be 'seen' in the brain. So taken was he with this problem that he pursued it through a doctoral thesis on how the neurosciences actually come to know specific disorders - a process that took the form of a gradual realization that the broadly social constructionist impetus motivating his project was quite useless for helping to understand the data it produced. As DF came to realize that the neurosciences were not what he thought they were, he found himself becoming increasingly disenchanted with the dominant explanatory logics of the social sciences as regards their accounts of psychiatric disorder and mental illness (for example, Martin 2004; Ortega and Vidal 2007). Through another chance recommendation from a graduate school colleague that he check out the literature on 'material feminism' (see Alaimo and Hekman 2008), and subsequent engagement with the work of cultural theorist Elizabeth Wilson (2004) and Donna Haraway (1997), one of the leading lights in science and technology studies (STS), DF came to think very differently about just what kind of practice cognitive neuroscience might actually $b e$ - and, in particular, about how that practice might get traced across the social and cultural environments in which it took place. The other author of this volume (FC), in the course of co-writing an article on the use of neuroscientific literatures within the humanities and social sciences (Papoulias and Callard 2010), became perturbed that she was not doing justice to the heterogeneity of these literatures - not least when she subsequently ended up at one of the heartlands of the psychiatric and neuroscientific establishment (the Institute of Psychiatry, Psychology \& Neuroscience (IoPPN), King's College London). The disjuncture between publically circulating accounts of cognitive neuroscience (which were often of the unsatisfying form: 'this bit of the brain lights up in these circumstances'), and the diverse and dynamic models, theories and vocabularies that many of FC's colleagues at the IoPPN mobilized, led her to pay much closer attention to how that space was being conjured.

For both of us, then, there was a significant drive to collaborate with neuroscientists in order to understand more about the specifics of ontologies, concepts, and also neuroscientific phenomena that felt quite alien to us. Compare this with how one of our neuroscientific collaborators, Simone Kühn (SK), explained why she engaged in an interdisciplinary study with the psychologists Russ Hurlburt and Charles Fernyhough. (We will discuss this collaboration in greater detail in Chapter 2.) 
SK: I was studying psychology [a while back]. I thought introspection was what psychologists should be interested in. That's why psychology is interesting: we want to know about inner experience. I found Russ [Hurlburt]'s publications and found his approach very, very interesting. I didn't know at that point that introspection was different from what Russ does [Descriptive Experience Sampling]. Now it's nice I can offer a [brain] scanner and my expertise and my neuroscientific methods.

What SK emphasizes is a long-standing interest in a particular problematic - the elicitation of inner experience - that led her to find research articles by the scientist with whom she later collaborated when she was more centrally embedded within neuroscientific than psychological research. For SK, the collaboration enabled her to bring her neuroscientific methods and expertise into contact with Russ Hurlburt's psychological methods for eliciting momentary states of consciousness; as she put it, 'I need certain skills that they [Russ and Charles] have; they need skills that I have.' Compare this with the account of another of our collaborators, medical humanities and cultural studies scholar, Angela Woods (AW):

$A W$ : I completed my doctorate - an analysis of schizophrenia in clinical and cultural theory - in a department of English with cultural studies, an environment all but fully insulated from clinical and scientific practice. All that changed immediately following my appointment to a philosophy-based lectureship in medical humanities in a school of medicine, pharmacy and health. Within a few weeks of arriving, the co-director of the Centre for Medical Humanities somewhat spontaneously invited me to a meeting with Charles Fernyhough and his doctoral student Simon McCarthy-Jones - psychologists interested in developing interdisciplinary approaches to the phenomenon of hearing voices. Eighteen months later, Charles, as Director, and I, as Co-Director, led a team of 13 co-applicants to secure substantial Wellcome Trust funding for the Hearing the Voice project (a large, interdisciplinary investigation of the experience of hearing a voice in the absence of any external stimuli); six years on, the collaboration continues to expand and develop - producing an ongoing upheaval in my own disciplinary identity along the way.

If, for SK, collaboration emerges from carefully considered opportunities to share methods, AW's account seems to us much more alive to serendipity, chance, and contingency. Moreover, AW's collaborative trajectory is not only tied to particular projects, but is also woven through an attention to a broader sense of a changeable disciplinary identity. Perhaps 
somewhere between these two approaches is the account of our collaborator Charles Fernyhough (CF), one of the psychologists mentioned above by both SK and AW:

CF: Returning to academia after a long break in the early 2000 , I realized that the experiences I was most interested in - inner speech, voice-hearing, memory - reached depths that standard psychological methodologies couldn't fathom. There was wisdom in the humanities that I had to try to tap, and my first experiments involved exhilarating collaborations on memory and space, mind and consciousness, thinking and feeling. As a humanist manqué, I found plenty to thrill me in, say, putting literary and psychological accounts of inner experience up against each other, and over time - particularly through the Hearing the Voice project and Hubbub - I've seen how this open-minded spirit of collaboration can spur scientists to raise their game in profound ways.

In CF's account we are inclined to hear both the orientation around specific questions and methods - but also a broader sense of his own variable trajectory, and an openness to where that might ultimately lead him.

Perhaps you, too, are - or have been - intrigued by conversations that you have overheard; or you've become bored with your own set of disciplinary debates. Perhaps you can no longer ignore the gap between what you've read about the neurosciences (or, indeed, other fields) in the canonical texts, and your own empirical encounters with those fields. Perhaps you think there are things about the materiality of human social life that are not exhausted by the canonical works of sociology and anthropology. Perhaps you can no longer face using certain scales in your scientific studies that you increasingly believe to have little face validity, and even less construct validity. If you're a social scientist, perhaps you just think that your social scientific and humanities colleagues who circle the neurosciences are more likely to get funding than you are. Perhaps you are a neuroscientist with a cosmopolitan approach to methods and theories. Perhaps you are wondering how you might gain access to resources specifically earmarked for interdisciplinary work. What actors, phenomena, and motivations, in other words, are present in your own landscape that might open up (or that have opened up) paths towards interdisciplinary collaboration? What might you do with your own, specific epistemological and affective orientations $v i s-\grave{a}$-vis the potential for interdisciplinary collaboration in these domains? 


\section{Getting started}

In many ways, we were both lucky to be in more or less the right place at the right time for the kinds of collaborative research that we wanted to do. Specifically, we benefited from two significant European networks and platforms that preceded us: the European Neuroscience and Society Network (ENSN; funded by the European Science Foundation (European Neuroscience and Society Network n.d.)) and the European Platform for Life Sciences, Mind Sciences, and the Humanities (funded and organized by the Volkswagen Foundation (VolkswagenStiftung n.d.)). These two platforms, both established in the mid-200os, were transformational in training and connecting new cohorts of researchers, fluent (or at least vaguely literate) in both the neurosciences and the social sciences. They were also both grounded in the conviction that physical co-location for a certain number of days is indispensable for sparking collaborative possibilities across the disciplines. And finally, beyond the format of the traditional workshop, both offered the opportunity for scientists and social scientists to work together to develop new experiments. (See Frazzetto 2011; Rose n.d. for illuminating accounts from two of the designers and originators of the residential ENSN Neuroschools, which include explications of the importance of engaging in 'experiment.) A little later, we were also both accepted on, and received funding to attend, the residential Neuroscience Boot Camp, at the Center for Neuroscience and Society at the University of Pennsylvania. (The nine-day boot camp is designed to provide academics, business people and policy makers with 'a basic foundation in cognitive and affective neuroscience and to equip them to be informed consumers of neuroscience research' (Center for Neuroscience \& Society, University of Pennsylvania 2015).) A confluence of temporary and contingent initiatives and institutions - none of which we participated in setting up or designing - was thus foundational in allowing us to acquire cross-disciplinary expertise, as well as meet collaborators from other disciplines.

It is worth stressing, again, that these networks represent a definite moment, in which there was a sense of flux around the relationship between the neurosciences and social sciences. The specificity of that moment is also evidenced by the emergence of a series of groups, coalitions, and interdisciplinary research arenas during roughly the same period - and to which our own forays are significantly indebted, both directly and indirectly. These include the NeuroGenderings Network 
(Dussauge and Kaiser 2012), the people and publications assembled around 'critical neuroscience' (e.g. Choudhury, Nagel, and Slaby 2009; Choudhury and Slaby 2012; Critical Neuroscience n.d.), the field of neuropsychoanalysis (Solms and Turnbull 2011; Fotopoulou, Pfaff and Conway 2012), the Association of Neuroesthetics (Association of Neuroesthetics 2015), the BIOS centre at the London School of Economics (BIOS 2015), as well as various 'Neurohumanities' programmes in prestigious universities (Hagouel 2012). We conjecture that that moment is no longer alive in quite the same way. It is certainly not that the avenues for interdisciplinary research have suddenly narrowed, but rather that the horizon of interdisciplinary possibility, in 2015-2016, is not quite the same as it was in, say, 20082009. Our hunch is that the sense of possibility that we witnessed has moved to other, currently 'sexier', biosocial fields. We think here, for example, of epigenetics (Landecker and Panofsky 2013) or metabolomics (Levin 2014). It is noteworthy that neither the ENSN nor the European Platform exists any longer (although a group of junior researchers, based primarily at King's College London, is attempting to resuscitate a 'Neuroscience and Society Network' - see Mahfoud and Maclean 2015). In our particular neck of the woods, it is noticeable that there now appear to be more large, grant-funded interdisciplinary projects that address the terrain of the brain and the mind (e.g. Hearing the Voice and Hubbub), and fewer specially designed 'platforms' or programmes to train cohorts of researchers competent in at least two disciplines (though note graduate programmes, such as the Berlin School of Mind and Brain, committed to training interdisciplinary researchers). None of which is at all to say that such opportunities are now entirely foreclosed - or that nothing remains for the would-be junior collaborator save the unenviable labour of shaving the rough edges from an earlier, open, precarious moment, now sadly passed. But there is a temporality, as well as a momentum, of openness and closure around specific interdisciplinary fields. For those interested in collaboration around the brain and mind, it's worth asking the question: What kind of moment are we actually in now in the relationship between the neurosciences and the social sciences? How might the specific dynamics and constellations of that moment shape how interdisciplinarity is imagined and practised? 


\section{Getting funded}

"I can't work out if they are actually going to do an imaging experiment, or just sit around and discuss it."

We do not overstate the case when we note that interdisciplinary funding applications have taught us more than anything else about the current shape of, and pressures on, interdisciplinarity across the social sciences and the neurosciences. All of the peer reviewer responses to grant applications that have accompanied us through the last few years have provided us with insights into both the scope and limits of the interdisciplinary field in which we have been struggling to find a place. We use fragmentary quotations from some of these comments throughout the book. We are aware that some will consider their use in a book - even, as here, without any marks of identification or attribution - to be in poor taste, or at least to run counter to the tacit norms through which such reviews are written and received within a restricted economy of circulation. The problem for us is that it is precisely in the construction and delivery of these reviews, understood here as perhaps the most potent and consequential genre of academic writing, that emergent fields, ideas, and problem-spaces are variously enacted, produced, realized, bounded, constrained, policed, squashed, and so on. We simply cannot write about the emergence of a new interdisciplinary field, still less narrate our own paths through that field, without some attention to moments of peer review. More broadly, and in excess of any other genre we have encountered, they stage the complexities, tensions, and excitements of 'interdisciplinarity', precisely at the moment in which interdisciplinarity inveigles itself into the strictures and assumptions of (to use a flat-footed term) 'normal science'.

There are two current certainties in the funding of interdisciplinary research: everyone wants to do it, and no one quite knows how. In the United Kingdom, national research councils - which are largely split along sharp disciplinary lines (such that there is an Arts \& Humanities Research Council (AHRC), a Medical Research Council, an Engineering and Physical Sciences Research Council, and so on) - are at pains to stress their keenness to fund work that falls between them (Research Councils UK n.d.). And even within individual research councils, there is a great desire to fund - and to be seen to fund - work that may traditionally 
fall outside the council's remit (see e.g. Economic and Social Research Council [ESRC] 2013; Arts \& Humanities Research Council 2015). A growing number of funders are prioritizing research that crosses the sciences, social sciences and humanities. In the United Kingdom alone, researchers seeking interdisciplinary funding might look to (this is a partial list that will likely already be out-dated by the time you are reading it): the AHRC's 'Science in Culture' scheme (Arts \& Humanities Research Council n.d.); the ESRC's 'Transforming Social Science' portfolio (Economic and Social Research Council 2013); the Medical Research Council's 'Skills Development Fellowships' (Medical Research Council n.d.); The Wellcome Trust's Hub award, Seed awards in both Science and the Medical Humanities, and Collaborative Awards (Wellcome Trust n.d.); and the Fellows Programme of MQ: Transforming Mental Health, a charity that funds mental health research (MQ n.d.) - to mention just a few. In Europe more broadly, scholars might look at the explicitly interdisciplinary imperatives of the European Commission's Horizon 2020 programme (European Commission n.d.), as well as opportunities provided by the Volkswagen Foundation (VolkswagenStiftung n.d.), and Brocher Foundation (Fondation Brocher n.d.), among others. In the United States, comparable opportunities are provided by the Interdisciplinary Behavioral and Social Sciences competition of the National Science Foundation (National Science Foundation 2015) or, more closely related to our interests, the Behavioral Science and Integrative Neuroscience research branch of the National Institute of Mental Health (National Institute of Mental Health 2015). With a view to finding one's way through this dispersed and varied assemblage, our pragmatic and uninspired advice is: persistence can pay off. We have had at least one interdisciplinary proposal turned down (very unambiguously) from one scheme, to be received more generously, and ultimately funded, by another. This, of course, is the story of research funding as such. But that degree of normal variation becomes, we think, much sharper in interdisciplinary contexts, when epistemological norms that might otherwise be taken for granted are often, suddenly, in question.

What, then, are some of the stumbling blocks? If the spirit of interdisciplinarity is willing in funders, the body often remains weak. Our own experience has been that interdisciplinary proposals - and here we do want to distinguish the inter- from the pretty standard multi-disciplinary - can have a tough time. Partly this is because peer review colleges and panels are often made up (not unreasonably) of people with strong 
disciplinary expertise. Thus a grant proposal crossing disciplines A and $\mathrm{B}$ is likely to be reviewed by an expert on A and an expert on B - neither of whom is necessarily likely to feel favourably towards the other, nor towards the interdisciplinary proposal as a whole. Below, in providing examples of peer reviewer comments we have received on some of our interdisciplinary grant applications, we dwell on what such comments might tell us about the possibility - or impossibility - of intervening in the field of interdisciplinary research on the mind and brain.

The quotation that begins this section comes from our first example. With some of our neuroscientific collaborators, we had proposed to run a series of brain-imaging experiments whose design would not be specified in advance, but which would emerge iteratively from a series of interdisciplinary workshops that we would run. This got short shrift: 'In my field (scientific and medical neuroimaging)', one reviewer wrote, 'it is imperative to describe the proposed experiment in detail, e.g. paradigms, number and type of subjects, analysis plan.... I did not feel sufficient information was provided to indicate the quality of outputs.' Here, we rub up against keenly guarded disciplinary norms surrounding robust research. But if this neuroscientist found our proposed experiment too loose and underspecified, another reviewer (likely someone from the social sciences) found it too concrete: our proposal demonstrated a 'naïve realism about the experiment and its ambitions for experimental convergence... [while the] ambition to establish a more general protocol to be followed by others is epistemically naïve and unreflexively normative'. The broader problem becomes clear here: try to design an interdisciplinary experiment that puts together the empirical rigour of neuroimaging and the conceptual openness of the humanities, and there is a good chance that you will be condemned by neuroscientists for your ambiguity, and by humanities scholars and/or interpretive social scientists for your empiricism.

In a second example - from a peer review of a different grant application - we were informed that while our project had 'potential', we should not only do more to test our proposed approach (presumably prior to applying, again, for funds), but specifically provide 'evidence of potential to engage neuroscientists in a reciprocal relationship. The reviewers did not see sufficient evidence of 'real collaboration with the community [we were] targeting. Note, first, how the injunction to engage our targeted community ('neuroscientists') betrays the assumed direction of travel: it seems inconceivable, for example, that the field might be too entangled 
to allow the identification of a 'target community'. The interdisciplinary endeavour, here - 'real collaboration' - is set out as an attempt by one discrete 'community' to target another in a reciprocal relationship. Indeed, this comment was offered in spite of the application's self-citation of articles co-authored with members of that same 'targeted community', some of whom were even co-applicants on that very grant. Our point, here, is not to offer a mundane gripe about peer review, but to note how reviewers might conjure interdisciplinary applicants as people desirous of a relationship with some other - and thus adjudicate them on the basis on whether or not they show evidence of their ability to so 'engage'. This is a very specific mode of interdisciplinarity - one that already forecloses a range of possibilities for crossing boundaries. What get potentially occluded here, are modes in which collaborators are already deeply intertwined with people who come from other disciplines, and in which the collaboration is embedded in tightly braided methodological and affective trajectories, rather than straight-laced strategies for targeting or engagement.

\section{Getting published}

Much could be written about the challenges of publishing for an interdisciplinary audience, given the pronounced differences in the cultures of publishing, the rhetorics at play, the formalities of presentation, differing conventions around citation, and so on. As with interdisciplinary grant proposals: write an article that tries to speak across the social sciences and neurosciences, and you might well be reviewed by a social scientist and a neuroscientist, either of whom might well object to what you're doing - but for different reasons. Below we summarize material from two peer reviews that we received in response to one interdisciplinary submission.

In the first, the reviewer, who explicitly identified him- or herself as a neuroscientist, noted that while s/he had found the manuscript very interesting, it was unlike anything s/he had read 'since [his/her] undergraduate women's studies courses'. Not only was the manuscript 'incredibly difficult to parse' - which s/he explained was a result of a 'stylistic difference across fields' - but it would, in its current form, be very unlikely 'to have any sort of impact upon neuroscientists or experimentalists'. (The reviewer carefully noted that there was no assumption that the authors 
wanted to have any such impact.) The advice? We should 'be much more direct about what it is that [we] mean to say'. Neuroscientists and experimentalists would, in fact, warm to 'a list of facts or statements separated by periods with the occasional transition term to hold it all together'; at the moment, it was hard to '[extract] the message out of what seemed like an artistically-written piece that made use of many unconventional phrases and secondary definitions of otherwise common words. The second reviewer came from quite a different angle - arguing that there was 'something peculiarly paradoxical in [the paper's] very concept'. We, the authors, were trying to set ourselves up as 'experimentally, experientially, and intellectually outside of the "black box", but in actual fact, we were held captive by 'the tools of cognitive neuroscience, i.e., on a scientific apparatus'. No matter the 'depth and authenticity of the joint collaborative exercise' for which we were advocating, we were pinioned by the inescapability and irreducibility of the scientific laboratory. For this reviewer, the point of 'this "fourth way" of neuro-engagement' was far from clear. For the scientist, the rhetorical style in which the 'artistically-written' paper was written obviated the chance that its arguments would be received by those coming from different, scientific, traditions of research writing. For the second reviewer, whom we assume to be a social scientist or humanities scholar, it conceded far too much to the sciences. This is a fairly consistent refrain. And yet, in general the two of us, as well as many of our collaborators, have been quite successful at publishing significantly interdisciplinary papers in relatively high impact journals - a success that we attribute at least in part to such papers having a certain currency among editorial board members at some journals. We do not want to prejudge whether this enthusiasm is necessarily shared by the editorial board members of many mainstream neuroscience journals. And for the collaborating neuroscientist, being published in a high impact social science journal, which may have both a relatively long time from submission to date of publication, and a relatively low impact factor' compared to even mid-ranking journals in some biological and medical fields, might represent poor return for her labour. (Let's note at this point that while the 2014 impact factor of the leading journal Nature Reviews Neuroscience was 31.427, that of Social Science \& Medicine (a very highly regarded social science journal) was 2.89o.)

There does appear to be a growing appetite among clinically-oriented journals for interdisciplinary research that crosses the life sciences, social sciences, and humanities. (e.g. The Hearing the Voice research team, of 
which one of us (FC) is a member, has had success in publishing in both Schizophrenia Bulletin (Woods et al. 2014) and Lancet Psychiatry (Woods et al. 2015). See also Woods 2015.) It is also noticeable that many of our own (and our collaborators') publications have been enabled by crossdisciplinary special issue calls (e.g. the special issue of Subjectivity on 'Neuroscience and Subjectivity' (see Cromby, Newton, and Williams 2011; Callard and Margulies 2011); the special topic on 'Critical Neuroscience' in Frontiers in Human Neuroscience (see Choudhury, Slaby, and Margulies 2015; Fitzgerald et al. 2014b; Callard and Margulies 2014) - and through new, more open and flexible spaces (again, see the Frontiers journals). New journals supporting interdisciplinary research are also being launched by reputable publishers (e.g. see Palgrave Communications). As with interdisciplinary grant applications, a certain degree of mobility and persistence - perhaps beyond the usual requirements of mono- or multi-disciplinary research - plays a major role in the publication of collaborative interdisciplinary papers.

\section{Tracking your process}

It is startling how few studies of interdisciplinarity in action there actually are, despite the almost daily injunctions for researchers to collaborate with people from other disciplines (and in striking contrast to the numerous processual and outcome-based evaluations of working practices that otherwise dominate the arts and sciences fields). On the one hand, this is a source of frustration for those keen to know more about such practices, as well as about the ontological stakes of such endeavours (e.g. see Greco 2013). On the other hand, it presents an opportunity: it means that researchers in interdisciplinary projects have access to data on an important and under-researched topic within the landscape of contemporary knowledge-production, viz. interdisciplinary collaboration.

We have attempted this kind of reflection previously (see e.g. Fitzgerald et al. 2014a), working mainly through auto-ethnographic reflection. In the course of our current work in Hubbub, however, we are trying to set out specific methods for capturing collaboration in action. One of us (DF) is keeping a field diary, both as a form of attention to his own current project - on how urban spaces get imagined, and intervened-upon, as locations of noise and restlessness - and more generally on the work of 'collaboration' in its own right, which is also one of the guiding logics of 
Hubbub itself. Focusing on Hubbub's different projects, the social interactions that make it up, its public statements, the strategies of its management team, and so on, this project resituates Hubbub within a broader field of early twenty-first century interdisciplinarity - and the projects within it as projects suspended within Hubbub's own collaborative logic. We are also using a qualitative and quantitative questionnaire (designed by Angela Woods, and drawing on her work in 'Hearing the Voice' (see Robson, Woods, and Fernyhough 2015 )), which is taking the pulse of the 50 or so collaborators in Hubbub at two different time-points. This questionnaire includes a social network analysis, which will be used to track the density and dynamics of the projects' collaborations over time - and which, incidentally, draws on our neuroscientific collaborators' expertise in topological methods that they employ in functional connectivity analyses of the brain (e.g. Margulies et al. 2013). We are also designing and trialling a new method called 'In the Diary Room' - which will be an automated collective reflection and self-tracking process, loosely inspired by 'The Diary Room' of Big Brother fame. Collaborators who volunteer will be randomly called into a small room just off our main project space, and will therein be asked a series of recorded, randomized questions about their day, how they feel the project is going, different feelings they have around collaboration in that project space, and so on. This is one of the ways in which we are gathering data about relations of power (see Chapter 6), and about the dynamics of affect (see Chapter 7 ). (For further details of all of these techniques, see Callard, Fitzgerald, and Woods 2015.)

The important point is that all the different elements of building an interdisciplinary career that we have discussed - finding collaborators, getting training, meeting people, applying for grants, having interdisciplinary papers published - are worth tracking and reflecting on in their own right. And this is not simply out of a narcissistic sense that one's own and one's collaborators' research trajectory is intrinsically fascinating, but rather because these mundane actions, spaces, and efforts make up what we might call, following Bruno Latour, the 'plasma' of interdisciplinarity, viz. the 'circulations of totalizations and participations' of interdisciplinary collaboration that are still 'waiting for explication and composition' $(2012,93)$. To put it more prosaically: if a range of interdisciplinary scholars begin tracking their own progress through these fields, and begin following the trajectories of the different people, things, ideas, bureaucracies, machines, and so on, that make them up - then not 
only might that progress, we conjecture, become more bearable; it might even help us all, collectively, to build a denser account of just what kind of interdisciplinary field it is, actually, that all those mundane actions and intra-actions are in the process of bringing into existence.

\section{Notes \& Queries: 1}

Q: What are some of the basic things out there that help someone who is potentially interested in doing some collaborative work across the disciplines? What are some of the things a person could do, or look out for, in terms of actually starting out and getting things done?

Outside of the major networks and funding initiatives that we have discussed in this chapter lay a whole series of other, small, mundane activities, which were, for us specifically, in many ways just as important in establishing the relationships and interests that either got us off the ground, or kept us going. And, as we have stressed here, we were fortunate to find ourselves within a place that had already been painstakingly carved out by many others before us - who first had the inkling that the separation of the biological from the social might lie on shaky ground. We also emphasize that it is often the gradual accumulation and assemblage of minor interventions that make up the nitty-gritty of interdisciplinarity, over and above the big grant calls, major co-authored papers, and so on. Below is a very partial list of things to look out for, which is culled from our own retrospective reflections:

- Residential workshops: Small, ephemeral residential workshops, with all the intersubjective intensity they entail, have been some of the most important locations for us, not only in terms of meeting people, but also for generating and sustaining a sense of excitement around particular questions: we have benefited especially from workshops funded by the European Science Foundation, the Volkswagen Foundation, and the Brocher Foundation.

-Visits and exchanges: For generating the bonds that make interdisciplinarity possible, as well as getting a better sense of the texture of an intellectual practice, there is no substitute for actually being present in someone's working space (whether a neuroscientific laboratory or a social sciences or humanities centre) 
for a while - not in the (sometimes) watchful, self-satisfied ethnographic mode, but rather through more open logics of hospitality and curiosity.

- Virtual spaces: Virtual spaces are vital for undergirding collaboration. They range from actual collaborative tools (such as Slack, Google Docs, or shared Zotero libraries), to simply having a decent Twitter presence that makes you visible to potential collaborators from other fields. Twitter is also good for keeping track of a field that isn't your own.

- Themed conference sessions: A lot of major conferences explicitly invite applications to curate themed sessions. These can be excellent opportunities for bringing potential interdisciplinary collaborators together.

- Taking courses: It was only through sitting in on a course on psychopathology that one of us learnt to take the neurosciences much more seriously. If you are a social scientist or humanities scholar, auditing a course on cognitive neuroscience can be enormously enlightening; if you are a neuroscientist, an exposure to what is actually entailed by qualitative methods might be extremely useful. You could even try out one of the Massive Open Online Courses (MOOCs). If you need really to get up to speed on the neurosciences, the University of Pennsylvania's residential Neuroscience Boot Camp is worth considering.

- Small grants: Many funders (and some institutions) offer 'seedcorn' or small grants to develop networks, or to pilot ideas. These can often be less competitive to acquire than larger grants, and can be an excellent means of bringing potential collaborators from across the disciplines together.

- Keeping up to date with journals and blogs: 'Collaboration' doesn't just mean working with someone, of course. You can adopt a collaborative stance simply by keeping up to date with the journals and blogs of another field - that is, knowing the major debates, the papers people are getting excited about, the controversies, and so on. 